OPEN

SUBJECT AREAS:

BIOMIMETIC SYNTHESIS

PHOTOCATALYSIS

VIRAL PROTEINS

NANOSTRUCTURES

Received

22 July 2013

Accepted

27 December 2013

Published

23 January 2014

Correspondence and requests for materials should be addressed to

A.G. (agupta@mint. va.edu) or P.E.P.

(prevelig@uab.edu)

\section{Formation mechanism of chalcogenide nanocrystals confined inside genetically engineered virus-like particles}

\author{
Ziyou Zhou ${ }^{1,2}$, Gregory J. Bedwell ${ }^{3}$, Rui Liं ${ }^{3}$, Peter E. Prevelige, Jr. ${ }^{3}$ \& Arunava Gupta ${ }^{1,2}$
}

\begin{abstract}
${ }^{1}$ Center for Materials for Information Technology, University of Alabama, Tuscaloosa, Alabama 35487, United States, ${ }^{2}$ Department of Chemical and Biological Engineering, University of Alabama, Tuscaloosa, Alabama 35487, United States, ${ }^{3}$ Department of Microbiology, University of Alabama at Birmingham, Birmingham, Alabama 35294, United States.
\end{abstract}

Engineered virus-like particles (VLP) are attractive for fabricating nanostructured materials for applications in diverse areas such as catalysis, drug delivery, biomedicine, composites, etc. Basic understanding of the interaction between the inorganic guest and biomolecular host is thus important for the controlled synthesis of inorganic nanoparticles inside VLP and rational assembly of ordered VLP-based hierarchical nanostructures. We have investigated in detail the formation mechanism and growth kinetics of semiconducting nanocrystals confined inside genetically engineered bacteriophage P22 VLP using semiconducting CdS as a prototypical example. The selective nucleation and growth of $\mathrm{CdS}$ at the engineered sites is found to be uniform during the early stage, followed by a more stochastic growth process. Furthermore, kinetic studies reveal that the presence of an engineered biotemplate helps in significantly retarding the reaction rate. These findings provide guidance for the controlled synthesis of a wide range of other inorganic materials confined inside VLP, and are of practical importance for the rational design of VLP-based hierarchical nanostuctures.

T he development of virus-based nanotechnology for biomedical, optical and magnetic applications is a growing field that aims to exploit their unique functional and structural properties ${ }^{1-5}$. In particular, the use of virus-like particles (VLP), which are composed of the capsid proteins lacking the viral genome and are non-infectious, has attracted increasing attention ${ }^{6,7}$. VLP assembled from protein subunits with precise 3D nanostructures exhibit a remarkable variety of shapes and structures, and can be produced in large quantities using biological amplification and growth. They are amenable to displaying foreign protein inserts through genetic engineering or chemical modifications ${ }^{8-10}$. Selective deposition of organic or inorganic materials, by design, at specific locations on the VLP affords precise control over the size, spacing, and assembly of nanomaterials, resulting in uniform and reproducible nano-architectures ${ }^{11-15}$. Both the exterior and interior surfaces of the VLP can be functionalized by genetically or chemically modifying the protein subunit ${ }^{16-18}$. For the exterior shell and inner cavity, site-selective polymerization, enzyme encapsulation and growth of inorganic materials have been demonstrated for a number of systems ${ }^{15,16,19-25}$. Such modifications can result in dramatic changes in the physicochemical properties, introduce new functionalities, or generate ordered nanostructures. Despite these advances, details regarding the mechanism of site-selective attachment, encapsulation and biomineralization kinetics remain largely unexplored.

Herein we report on the site-specific mineralization and growth behavior of semiconducting cadmium sulfide (CdS) nanocrystals confined inside bacteriophage P22 VLP, which have been shown to be a robust and versatile biotemplate for the synthesis of a range of ordered nanomaterials ${ }^{17,26-28}$. In particular, our efforts are aimed at elucidating and understanding the nucleation, growth and kinetics of CdS formation inside the VLP nanocages. Our study provides valuable insights into biomineralization mechanism for confined growth inside engineered virus-like nanoreactors, and is potentially relevant for a wider range of inorganic materials.

The P22 VLP are assembled from 420 identical copies of coat proteins (CP) and approximately 300 copies of scaffolding proteins (SP), with a diameter of around $60 \mathrm{~nm}^{27}$. Upon heating, structural transitions of P22 yield VLP with larger internal capacity and enhanced external permeability to the hollow cavity ${ }^{17,29}$.

To generate CdS binding VLP the peptide sequence SLTPLTTSHLRS, isolated by Belcher's group through screening of M13 phage display library and confirmed to have binding specificity to CdS, was genetically fused onto the $\mathrm{SP}^{30,31}$. The SP are expected to be symmetrically localized in the interior of the viral cavity ${ }^{32,33}$. 


\section{Results}

In a typical synthesis, $0.1 \mathrm{mg}$ of genetically engineered P22 procapsid-like particles (EPC) was dispersed in $1 \mathrm{~mL}$ aqueous solution of $\mathrm{CdCl}_{2}(0.2 \mathrm{mM})$ at room temperature for $4 \mathrm{~h}$, allowing for protein$\mathrm{Cd}^{2+}$ interaction. Subsequently, $2 \mathrm{uL}$ of aqueous thioacetamide (TA, $0.1 \mathrm{M}$ ) was added to the solution and the mixture maintained at room temperature for an additional hour. The hydrolysis of TA, which results in the release of sulphur ions, was initiated by adding an aqueous solution $(25 \mathrm{mM})$ of $20 \mathrm{uL}$ tris(hydroxymethyl)aminomethane (Tris). The $\mathrm{pH}$ of the Tris solution was adjusted to $9.5 \mathrm{using}$ $\mathrm{NaOH}(4 \mathrm{M})$. We chose TA as the sulphur source since the release of sulphur can be suitably controlled by hydrolysis and, more importantly, its decay kinetics can be monitored in-situ by UV-Vis spectroscopy, as discussed later. For comparison, CdS was also synthesized using only the engineered scaffolding proteins (ESP) in solution. In addition, control experiments were carried out using wild-type coat proteins (WCP), or just in DI water without any proteins.

To confirm that we have successfully confined the growth of CdS inside EPC, the EPC/CdS nanostructures were negatively stained with uranyl acetate and observed using the STEM (Scanning Transmission Electron Microscopy) mode of a JOEL 7000 SEM (Scanning Electron Microscope). The clear presence of core/shell structures in Figure 1a confirms the confined growth of CdS inside the P22 procapsids. The observed contrast in the core/shell images can be attributed to the combined outcome of negatively stained P22 coat proteins and the CdS packed in the interior. The average diameter of twenty randomly selected shells and cores is determined to be $67.1 \mathrm{~nm}$ and $40.6 \mathrm{~nm}$, respectively (size distribution shown as Figure $1 b$ ). These values are similar to the known external and internal diameters of P22 procapsids ${ }^{17,34}$. We also imaged the unstained CdS-EPC nanostructures using STEM, as shown in supplementary figure S1. At 30,000 $\times$ magnification, uniform CdS nanostructures with average size of around $40 \mathrm{~nm}$ are observed, which is in accordance with the core size distribution in Figure $1 \mathrm{~b}$. At the same magnification, the images for unstained CdS-EPC nanostructures are less sharp and smaller in size than those of stained CdS-EPC (supplementary figure S2). This can be attributed to the fact that the P22 coat protein composed of non-conductive and light elements does not provide adequate contrast. Comparison of the images indicates that the growth of CdS occurs exclusively inside the P22 procapsids, and the outside coat proteins are left intact.

The confined growth of CdS inside P22 VLP is also confirmed from TEM (Transmission Electron Microscopy) images. The size of unstained CdS/VLP shown in supplementary figure S3a is around $40 \mathrm{~nm}$, which is much smaller than the size of stained P22 VLP shown in supplementary figure $\mathrm{S} 3 \mathrm{~b}$. This result is in agreement with the STEM results shown in Figure 1. The HRTEM (High Resolution TEM) image of a single nanostructure further confirms that the crystal phase of CdS is hexagonal, with the characteristic lattice fringes clearly observed as shown in supplementary figure S4a. The SAED pattern of the CdS nanostructures over a larger area, shown in supplementary figure $S 4 b$, is also consistent with the hexagonal wurtzite structure of CdS with polycrystalline characteristics because of the random orientation of the particles. These results are in agreement with the results reported previously for CdS growth by Belcher's group and our group ${ }^{28,35}$.

To further confirm that the growth of CdS occurs inside the P22 VLP, we carried out dynamic light scattering (DLS) measurements to obtain the hydrodynamic size before and after growth, as shown in supplementary figure S5. The average hydrodynamic size of EPCs in solution is determined to be $68 \mathrm{~nm}$. After the growth of nanocrystals for $24 \mathrm{~h}$, the average size remains essentially unchanged, with a somewhat narrower distribution. The DLS and electron microscopy results conclusively demonstrate that the coat protein shells confine the growth of CdS nanocrystals.
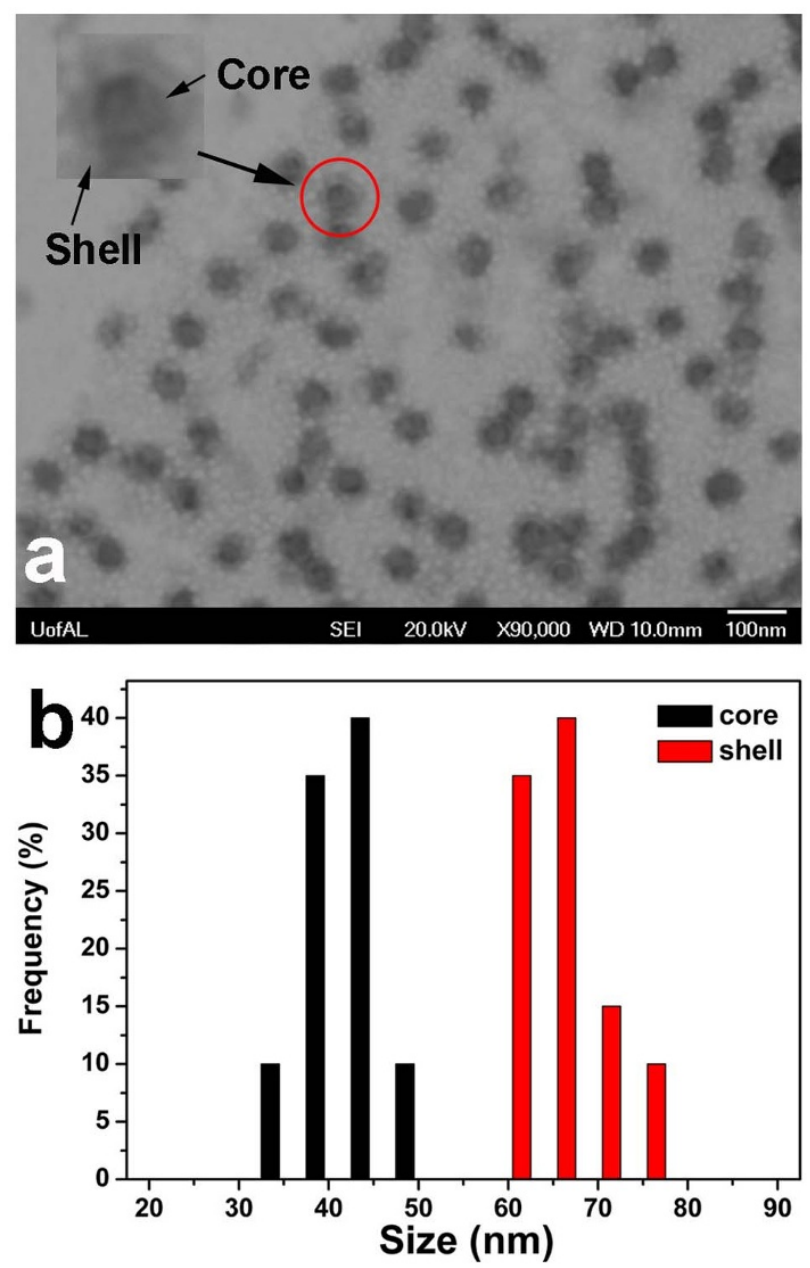

Figure $1 \mid$ (a) STEM image of the growth of CdS constrained inside genetically engineered $\mathrm{P} 22$ procapsids, stained with uranyl acetate; and (b) size distribution of P22 shells and CdS cores based on 20 randomly selected particles from Fig. 1a.

Interestingly, the confined growth does not ultimately result in a dense core of CdS inside P22 VLP. While the slow growth of CdS inside EPC continues with increasing reaction time, nowhere close to complete packing of the EPC interior is achieved even after an extended period. We have incubated the reaction solution for up to a week with excess concentration of the precursors and observed that the EPC break up instead of forming a dense inorganic core (supplementary figure S6). Possible reasons for the rupture of P22 VLP are discussed later.

To better understand the biotemplated growth process of CdS confined inside P22 VLP, we carried out time-dependent TEM studies to monitor the growth. The results indicate that the formation of $\mathrm{CdS}$ involves uniform nucleation and growth during the initial stage, followed by stochastic growth for longer reactions periods. The protein-initiated nucleation of CdS nanocrystals appears to occur at the genetically engineered peptide sequences of the scaffolding proteins, which is experimentally determined from sedimentation velocity experiments using Analytical Ultracentrifugation (AUC) to be approximately 300 copies per procapsid (the detailed method and data analysis can be found in Methods and Supplementary Information (supplementary figure S7)). Ideally, the ESP are expected to be uniformly distributed inside the VLP cavity, which implies that there will be roughly 300 evenly distributed nucleation sites. Based on the TEM image in Figure 2 a1, we indeed observe fairly uniform nucleation and growth of CdS during the early reac- 
tion period $(1 \mathrm{~h})$. Small nanocrystals with an average size of $2.5 \mathrm{~nm}$, as estimated from 20 randomly selected assemblies, are uniformly distributed inside the P22 shell (with the size distribution shown in supplementary figure S8). The total number of nanocrystals is counted to be 205 (marked in Figure 2 a2, using imageJ software), which is in the expected range of 150-300 that are expected to be observed if 300 points are projected onto a plane. The size of the nanostructures progressively increases after 3 hours and 10 hours of reaction (Figure $2 \mathrm{~b} 1$ and Figure $2 \mathrm{cl}$ ) and has a broader distribution, with an average size of 5.4 and $5.8 \mathrm{~nm}$, respectively (supplementary figure S7). Correspondingly, the number of isolated nanostructures are counted to be 59 (Figure 2 b2) and 36 (Figure 2 c2). These values are far less than what is expected for continued uniform growth of the nanocrystals. This, coupled with the observed broadening of the size distribution, indicates that the latter stages of $\mathrm{CdS}$ growth occur more or less randomly. To further confirm this conclusion, we carried out size-dependent simulations assuming that the individual CdS subunits grow uniformly inside the spherical nanocage (see methods for details). With a size of $2.5 \mathrm{~nm}$ for the CdS assemblies, the simulated result matches well with the observed TEM result, as shown in Figure 2 a3. However, with simulated sizes of $5.4 \mathrm{~nm}$ and $5.8 \mathrm{~nm}$, the results are very different from what are actually observed. We can thus conclude that the initial nucleation and growth of CdS is uniform and evolves to much more random growth for longer reaction periods.

The nature of CdS growth can be attributed to the fact that the distribution of engineered scaffolding proteins is limited by the volume of inner space of P22 VLP. During the initial stage there is sufficient distance between SP for uniform nucleation and growth of $\mathrm{CdS}$ nanocrystals to occur at the genetically engineered sites of the scaffolding proteins. As the size of individual nanocrystals increases, there is increased likelihood for adjacent CdS/SP to coalesce together. Because of the flexible nature of scaffolding proteins in solution ${ }^{36,37}$, the fusion of CdS/SP is stochastic resulting in random formation of larger clusters. This phenomenon may also explain why complete packing of CdS cannot be achieved inside P22 VLP. The solubility product $\left(\mathrm{K}_{\mathrm{sp}}\right)$ for CdS in water is extremely low at room temperature $\left(\sim 1 \times 10^{-27}\right)$, resulting in fast nucleation of the nanocrystals at the template surface as soon as both sulphur and cadmium precursors are available ${ }^{38,39}$. However, the scaffolding proteins are electrostati-
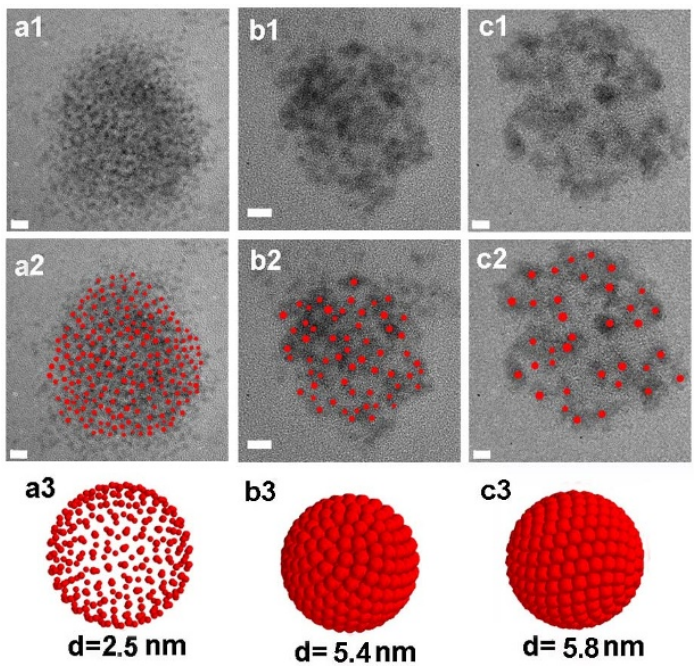

Figure 2 TEM images of CdS after growth time of (a1) $1 \mathrm{~h}$; (b1) $3 \mathrm{~h}$; and (c1) $10 \mathrm{~h}$. Counts of CdS nanostructure subunits after growth of (a2) $1 \mathrm{~h}$; (b2) $3 \mathrm{~h}$; and (c3) $10 \mathrm{~h}$ and size-dependent simulation of CdS nanostructure subunits with size: (a3) $2.5 \mathrm{~nm}$; (b3) $5.4 \mathrm{~nm}$; and (c3) $5.8 \mathrm{~nm}$. Scale bars for a1, a2, c1, and c2 represent $5 \mathrm{~nm}$, and for b1 and b2 represent $10 \mathrm{~nm}$. The TEM images are obtained without any staining. cally attached to the inner shell of coat proteins ${ }^{40,41}$, which restrains the diffusion of mineralized SP/CdS into the central cavity of P22 VLP and results in the growth being primarily restricted to the interfacial region between the SP and shell of P22 VLP. The combined effects of continuous rapid nucleation and restrained diffusion results in large CdS nanoclusters randomly formed between the SP and shell of VLP. As the size of CdS nanoclusters increases, the shell becomes increasingly unstable and eventually results in the rupture of P22 VLP.

For comparison, we also studied the growth of CdS using only the ESP as templates for reaction times of 1 and 3 hours, as shown in supplementary figure S9. After $1 \mathrm{~h}$, the size of CdS nanocrystals is around $3 \mathrm{~nm}$, which is in accordance with the size of CdS grown inside EPC. After $3 \mathrm{~h}$ of reaction, the CdS nanocrystals self-assemble to form nanoflower-like clusters with size between 15-20 nm, which is much larger than what is observed inside the procapsids. These results further confirm that the coat proteins effectively constrain and restrict the growth of CdS inside the P22 VLP. The crystal structure of CdS has been further investigated by high resolution TEM (HRTEM), as shown in the insets of supplementary figure S9. Lattice fringes at both time $1 \mathrm{~h}$ and $3 \mathrm{~h}$ can be indexed to the (100) planes of hexagonal CdS. This is in agreement with the SAED result. The clustered structure of CdS synthesized by biotemplates, with or without the presence of coat proteins, suggests that the nucleation of CdS adopts a non-classical pathway, as also evidenced in previous mineralization studies of inorganic materials such as calcium carbonate and calcium phosphate using biotemplates ${ }^{42-46}$.

We have also investigated the growth of CdS particles using wildtype P22. In this case, CdS growth occurs only in solution in the form of spherical nanoparticles with an average size around $10 \mathrm{~nm}$, and not inside the VLP, as shown in supplementary figure S10. Some large particles are also observed with size between $60-80 \mathrm{~nm}$, which may results from non-specific growth of CdS on the outside layer of P22 coat proteins.

To probe the reaction kinetics, we have monitored in-situ the formation of CdS inside EPC using UV-Vis spectroscopy. The absorbance intensity at $260 \mathrm{~nm}$ - the characteristic absorption peak of TA - is well-suited for simultaneously detecting changes in the concentration of the sulphur source. As seen in Figure 3, the TA absorbance decreases as expected with increasing reaction time, while the absorbance in the range of 400-500 $\mathrm{nm}$ due to CdS increases. The absorption variations for CdS as a function of wavelength for different reaction durations are shown on an expanded scale as an inset in Figure 3. A broad absorption feature lacking defined peaks, with a

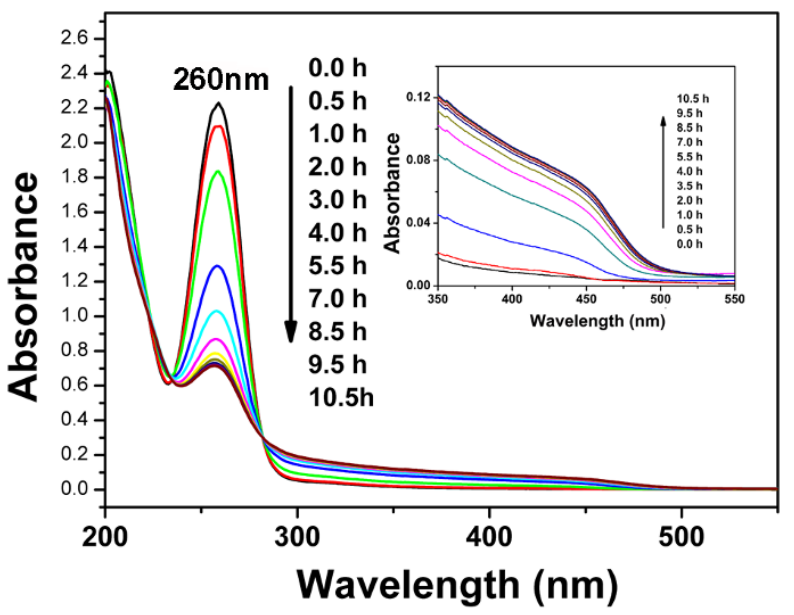

Figure 3 In-situ observation of the growth kinetics of CdS by UV-Vis spectroscopy from monitoring changes in the absorbance of TA and CdS. The inset shows a magnified plot of the absorbance variation in the range of 550-350 nm due to changes in CdS concentration. 
cut-off at longer wavelength, is noted for the absorption of CdS. The absorption shoulder systematically shifts to longer wavelengths with increasing duration of TA hydrolysis. This shift in the absorption shoulder is rapid during the first few hours and then slows for longer reaction periods. This suggests a rapid change in size during the early stage of CdS growth, and a slower size increase for longer reaction periods, which is consistent with the increase in the average size of the nanostructures observed from the TEM images in Figure 2. Using Henglein's empirical equation ${ }^{47,48}$, we have calculated the band gap and size of the CdS nanostructures from the absorption edges, as shown in Figure 4 and supplementary Table S1. The band gap decreases from $2.70 \mathrm{eV}$ for reaction time of 1 hour to $2.53 \mathrm{eV}$ for 3 hours and eventually to $2.48 \mathrm{eV}$ for reaction times longer than 8 hours. The steady decrease of the band gap indicates a corresponding increase of CdS size with reaction time. Based on Henglein's equation, the average size is determined to be $3.85 \mathrm{~nm}$ after reaction time of 1 hour and $6.04 \mathrm{~nm}$ after reaction time longer than 8 hours. Overall, the results agree well with those determined by TEM for longer time periods, as shown in Figure 4. However, there is a discrepancy in the size of CdS determined from TEM and that from UV/ Vis measurements for the shortest reaction period (1 hour). Previous studies have shown that CdS nanocrystals of very small size exhibit strong interaction with aromatic groups, resulting in a red-shift of the absorption edge ${ }^{49,50}$. In the CdS-specific binding peptide SLTPLTTSHLRS, the aromatic ring in histidine $(\mathrm{H})$ can likely interact with the nanocrystals during the early stages of nucleation and growth, causing a red-shift of the absorption edge and thus an increase in the estimated size using Henlein's equation.

As expected, the integrated absorption intensity of the broad absorption shown in Figure 3 increases with reaction time, indicating an increase in the CdS concentration ${ }^{48,51}$. As a rough estimate of product formation from the reactants, we have calculated the consumption of TA and the generation of CdS at different time intervals. The trend of TA consumption matches well with that of CdS generation, as shown in supplementary figure S11.

We have also investigated the TA hydrolysis kinetics under different reaction conditions, and the results are summarized in Figure 5. TA has a characteristic absorption peak at $260 \mathrm{~nm}$, which enables us to precisely monitor the decrease of TA concentration during hydrolysis. However, the $\mathrm{P} 22$ proteins and CdS nanoparticles also absorb at $260 \mathrm{~nm}$. To obtain accurate TA concentration, we used Nanosep ${ }^{\circledR}$ centrifugal filter devices (Pall Life Sciences, MWCO 3000) to remove any species in reaction solution with molecular weight larger than

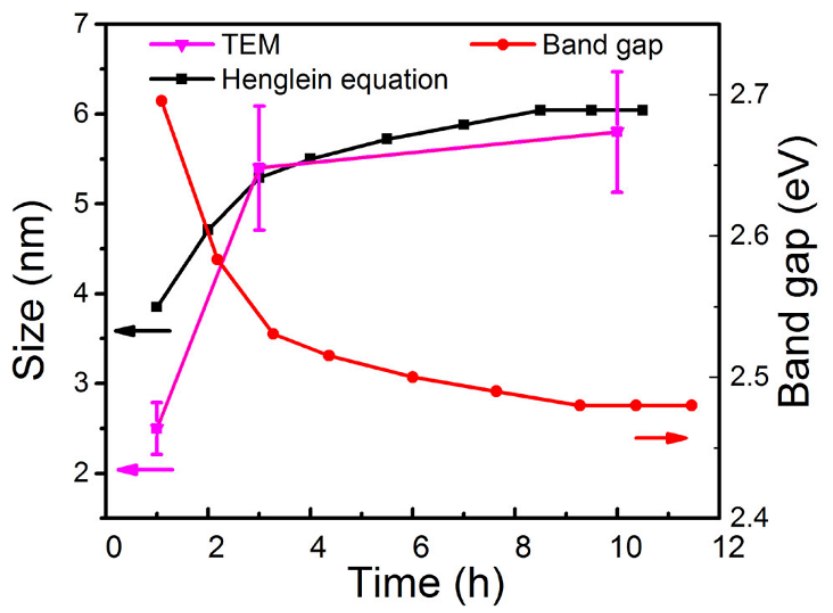

Figure 4 Size and band gap of CdS nanoparticles as a function of reaction time. The size of nanoparticles obtained directly from TEM measurements by randomly selecting 20 particles $(n=20$, error bars represent \pm S.D.) and those estimated from the absorption edge using Henglein's equation are shown in the figure.

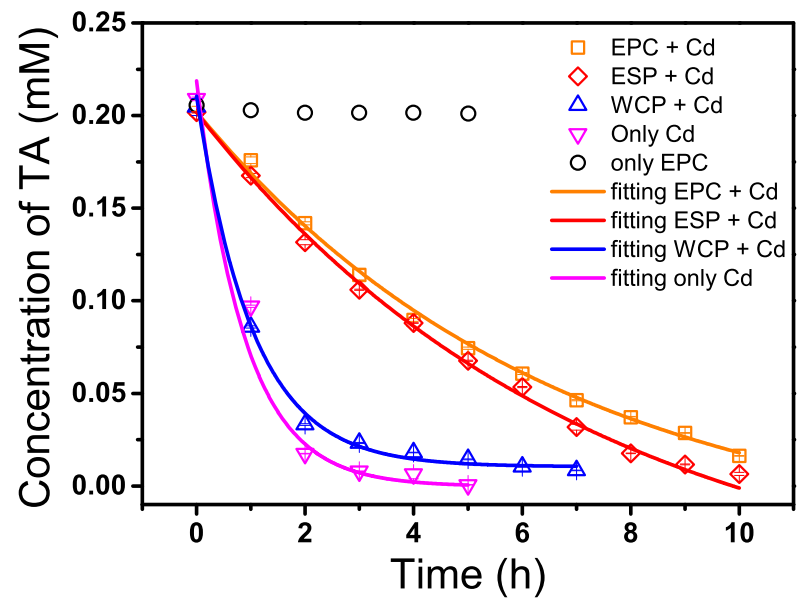

Figure 5 UV kinetic studies for the hydrolysis of TA (a) with EPC but without $\mathrm{CdCl}_{2}$; (b) with both $\mathrm{EPC}$ and $\mathrm{CdCl}_{2}$, corresponding fitting: $\mathrm{C}_{\mathrm{TA}}$, EPC $=0.22 * \exp (-t / 6.43), \mathrm{R}^{2}=0.991 ;(c)$ with both $\mathrm{ESP}$ and $\mathrm{CdCl}_{2}$, corresponding fitting: $\mathrm{C}_{\mathrm{TA}, \mathrm{ESP}}=0.23 * \exp (-\mathrm{t} / 5.95), \mathrm{R}^{2}=0.995$; and $(\mathrm{d})$ with both WCP and $\mathrm{CdCl}_{2}$, corresponding fitting: $\mathrm{C}_{\mathrm{TA}}$, wCP $=$ $0.20 * \exp (-\mathrm{t} / 1.03), \mathrm{R}^{2}=0.943$; (e) with just $\mathrm{CdCl}_{2}$ but without protein, corresponding fitting: $\mathrm{C}_{\mathrm{TA}, \mathrm{DI}}=0.21 * \exp (-\mathrm{t} / 0.88), \mathrm{R}^{2}=0.915$. EPC: engineered P22 procapsid, ESP: engineered scaffolding protein, WCP: wild-type coat protein. ( $\mathrm{n}=3$ independent experiments, error bars represent \pm S.D).

3000. Without any Cd ions there is no consumption of $\mathrm{S}^{2-}$ and the TA concentration remains essentially unchanged in EPC solution. In the presence of Cdions, $\mathrm{S}^{2-}$ is consumed to form $\mathrm{CdS}$, thus favoring further dissociation of $\mathrm{TA}^{52}$. Interestingly, as compared to the biotemplated synthesis of CdS, a much faster decrease in the TA concentration is observed in aqueous solution of $\mathrm{Cd}^{2+}$ without a biotemplate.

For all the different reactions the experimentally observed decrease in the TA concentration with time can be fitted well by an exponential, as shown in Figure 5. Based on fitting results, the decrease in TA concentration for all the reactions follows a first order reaction kinetics. For the hydrolysis of TA without any protein, the rate constant $k_{1}$ is $\sim 1.1 \mathrm{~h}^{-1}$, whereas for the hydrolysis of TA in the presence of WCP, the rate constant $k_{2}$ is $\sim 1.0 \mathrm{~h}^{-1}$. With just the ESP, the rate constant $k_{3}$ is $\sim 0.17 \mathrm{~h}^{-1}$, and with EPC the rate constant $k_{4}$ is $\sim 0.15 \mathrm{~h}^{-1}$. As compared to the case without engineered biotemplates or wild-type coat proteins, the rate constant is significantly lower for the consumption of TA in the presence of engineered proteins, as shown in supplementary figure S12. We also studied the TA hydrolysis rate as a function of EPC concentration, as shown in supplementary figure S13. The hydrolysis rate decreases with increasing concentration of EPC. Considering that wild type coat proteins do not appreciably affect the reaction kinetics as compared to TA hydrolysis without any protein, and the EPC concentrationdependent behavior of TA hydrolysis, we conclude that the specific CdS-binding peptides play a critical role in slowing the reaction kinetics. In the absence of an engineered biotemplate, other than weak interaction with wild type protein or Tris molecules, Cd ions in solution can combine freely with sulphur ions released from hydrolysis of TA and precipitate out $\mathrm{CdS}^{53}$. However, in the presence of an engineered biotemplate most of the CdS clusters formed immediately after binding of $\mathrm{Cd}$ ions with $\mathrm{S}$ ions are likely bound and stabilized by the genetically engineered scaffolding proteins, which blocks some of the active growth sites of the CdS nuclei. This slows down the growth of $\mathrm{CdS}$ and impedes the reaction equilibrium for its formation, thus retarding the hydrolysis of TA and thereby influencing the final hydrolysis equilibrium. The observed behavior of retarded CdS biomineralization is analogous to that of $\mathrm{CaCO}_{3}$ 
mineralization, which can be appropriately controlled by using various additives to regulate its reaction rate $\mathrm{r}^{54-57}$.

We have also carried out photoluminescence (PL) measurement of CdS grown inside P22 VLP after 10 hours, as shown in supplementary figure S14. A broad PL spectrum is obtained in the range of $450 \mathrm{~nm}$ to $650 \mathrm{~nm}$ with a peak around $537 \mathrm{~nm}$. The broad spectrum is consistent with the UV/Vis spectrum lacking defined peaks, which suggests a broad size distribution of CdS nanoclusters inside P22 VLP. The emission peak position around $537 \mathrm{~nm}$ is also in agreement with absorption edge values determined from the UV/Vis spectra (shown in supplementary Table S1). The UV/Vis and PL results suggest that the CdS nanostructures may be useful as visible light photocatalyst $^{58}$ or bioimaging agent ${ }^{59,60}$.

\section{Discussion}

Our results clearly demonstrate that genetically engineered scaffolding proteins evenly positioned inside bacteriophage P22 VLP can act as selective biotemplates for the constrained growth of CdS nanocrystals. The formation of CdS confined inside the VLP involves initial uniform nucleation and growth at the genetically engineered sites of the scaffolding proteins, followed by a more stochastic growth for longer reaction periods. The presence of the biotemplates does not affect the reaction order for the formation of CdS, but significantly influences the rate constant for the hydrolysis of thioacetamide (TA) and subsequent reaction of released $\mathrm{S}^{2-}$ ions with $\mathrm{Cd}^{2+}$. These findings provide important clues for the rational synthesis of chalcogenide and other inorganic nanocrystals confined inside VLP. For example, controlled synthesis of CdS nanocrystals with size around $3 \mathrm{~nm}$ inside P22 VLP can be achieved by simply centrifuge filtering the solution and terminating the reaction at time 1 hour. Additionally, the concentration of genetically engineered biotemplates can be adjusted to control the reaction kinetics. Understanding the formation mechanism and reaction kinetics further provides guidance for the future rational design of VLP-based hierarchical nanostructures. For constructing core/shell nanostructures using genetically engineered VLP as platform, e.g., synthesizing a nanostructured inorganic material confined inside VLP and a different material on the shell of VLP, our findings offer pathways for controlling the inner core structure.

\section{Methods}

Materials. CdS was synthesized using commercially available reagents. Cadmium chloride $\left(\mathrm{CdCl}_{2}\right)$ and thioacetamide $\left(\mathrm{C}_{2} \mathrm{H}_{5} \mathrm{NS}\right)$ were purchased from Acros Organics (New Jersey, US). Sodium Hydroxide $(\mathrm{NaOH})$ was purchased from Fisher Scientific (Pennsylvania, US), and Tris(hydroxymethyl)aminomethane (Tris) was purchased from VWR (Pennsylvania, US). All the chemicals were used as-received without any further purification.

Plasmids construction. Peptide sequence, SLTPLTTSHLRS, was added to the Nterminus of the truncated scaffolding protein by introducing the peptide coding sequencing (pCdS) into the $5^{\prime}$ end of the truncated scaffolding gene (gp8-141-303). We designed primers: 1) 5'-GCTGACCACCAGCCATCTGCGTAGCGCTCG CAGCAATGCCGTAGC-3'; 2) 5' -GACGATGCATATGAGCCTGACCCCGCTGA CCACCAGCCATCTGCGTAGC-3'; 3) 5' -TGGATCCTATTATCGGATTCCT TTAAG- $3^{\prime}$, to which peptide coding sequencing (AGCCTGACCCCGCTGACCA CCAGCCATCTGCGTAGC) was included. Using the plasmid of P22 gp8-141-303pET3a (from Dr. Prevelige's lab) as a template, the fragment of pCds-gp8-141-303 with the restriction enzyme sites NdeI and BamHI was obtained by two-step PCR, then digested and ligated into P22 assembly plasmid gp8. 5-pET3a with the same restriction enzyme sites was used to create the new assembly plasmid pCds-gp8-141304-gp5-pET3a.

Protein expression. The P22 coat protein and truncated scaffolding protein were heterologously expressed in BL21 (DE3) cells. BL21 cells containing the assembly plasmid pCds-gp8-141-304-gp5-pET3a were grown in $1.5 \mathrm{~L}$ of LB at $37^{\circ} \mathrm{C}$. Upon reaching mid-log phase, expression of protein was induced with the addition of $1 \mathrm{mM}$ IPTG. Induction was allowed to proceed for 3 hours before centrifugation at $9000 \times \mathrm{g}$. The cell pellets were then re-suspended in lysis buffer $(50 \mathrm{mM}$ Tris- $\mathrm{Cl} \mathrm{pH} \mathrm{7.6;}$ $100 \mathrm{mM} \mathrm{NaCl} ; 2 \mathrm{mM} \mathrm{MgSO}_{4}$ ) and frozen. Cells were lysed by freeze-thaw and sonicated to reduce the viscosity of the re-suspension. Following sonication, the resuspension was centrifuged for 45 minutes at $27,000 \times \mathrm{g}$ to clear cell debris. The soluble material was then centrifuged through a $20 \%$ sucrose cushion for $2 \mathrm{~h}$ at
40,000 rpm and $4{ }^{\circ} \mathrm{C}$ in a 42.1 rotor (Beckman Coulter). The pellet was re-suspended and the particles were loaded onto a 5-20\% sucrose gradient and centrifuged in an SW41 rotor (Beckman Coulter) for 30 minutes at $20^{\circ} \mathrm{C}$. Procapsid-like particles were found to migrate near the center of the gradient. The particles were collected and dialyzed against Buffer $\mathrm{B}(50 \mathrm{mM}$ Tris- $\mathrm{Cl} \mathrm{pH} \mathrm{7.6;25} \mathrm{mM} \mathrm{NaCl})$ and then stored at $4^{\circ} \mathrm{C}$.

The scaffolding protein construct was expressed and lysed in a manner identical to the procedure used for procapsid-like particles. Following cell lysis, the cell debris was separated from the soluble material by centrifugation at $27,000 \times \mathrm{g}$ for $1 \mathrm{~h}$. Ammonium sulfate was then added to the supernatant at $50 \%$ saturation. The pellet obtained after the $50 \%$ salt cut was re-suspended in 10-20 mL Buffer B and dialyzed extensively against Buffer B. The soluble material was then loaded onto $5 \mathrm{~mL}$ HiTrap Q and HiTrap SP columns (GE Healthcare) connected in tandem and the loaded material was washed extensively with Buffer B. The HiTrap Q column was removed and elution from the SP column was achieved by applying a linear gradient from $25 \mathrm{mM}$ to $400 \mathrm{mM} \mathrm{NaCl}$. Fractions containing scaffolding protein were pooled and concentrated using 10,000 Da MWCO Centricon centrifugal filter units (Millipore).

Inorganic synthesis. Genetically engineered bacteriophage P22 virus-like particles (EPC) stored in storage buffer were $4,000 \times$ dialyzed against deionized water before being used as biotemplates. In a typical synthesis, $0.1 \mathrm{mg}$ EPC was dispersed in $1 \mathrm{~mL}$ aqueous solution of $\mathrm{CdCl}_{2}(0.2 \mathrm{mM})$ at room temperature for $4 \mathrm{~h}$, allowing for protein- $\mathrm{Cd}^{2+}$ interaction. Subsequently, $2 \mathrm{uL}$ of aqueous thioacetamide (TA, $0.1 \mathrm{M}$ ) was added to the solution and the mixture maintained at room temperature for an additional hour. The hydrolysis of TA, which results in the release of sulphur ions, was initiated by adding an aqueous solution $(25 \mathrm{mM})$ of $20 \mathrm{uL}$

tris(hydroxymethyl)aminomethane (Tris). The $\mathrm{pH}$ of the Tris solution was adjusted to 9.5 using $\mathrm{NaOH}(4 \mathrm{M})$. For comparison, CdS was also synthesized using only the engineered scaffolding proteins (ESP) in solution. In addition, control experiments were carried out using wild-type coat proteins (WCP), or just in DI water without any proteins. All the reported experimental procedures were carried out in a fume hood with appropriate handling of the chemicals and biological samples for safety.

Materials characterization. The morphology and structure of the products were observed using the STEM (Scanning transmission electron microscopy) mode of a JEOL 7000 Scanning Electron Microscopy (SEM) and by Transmission Electron Microscopy (TEM) coupled with high resolution (HR) (Tecnai F-20). 2\% uranyl acetate was used as a negative staining agent. The hydrodynamic size of P22 procapsids and P22-CdS in aqueous solution was determined using a Zetasizer nano series dynamic light scattering (DLS). The SP content in P22 VLP was determined by sedimentation velocity experiments using a Beckman XL-A ultracentrifuge at $280 \mathrm{~nm}$ and $20^{\circ} \mathrm{C}$. The optical measurements were carried out using a UV-Vis spectrometer (Beckman DU 800) and an ISS PC1 photon-counting spectrofluorometer with excitation wavelength of $350 \mathrm{~nm}$.

1. Douglas, T. \& Young, M. Viruses: Making friends with old foes. Science 312, 873-875 (2006)

2. Douglas, T. \& Young, M. Host-guest encapsulation of materials by assembled virus protein cages. Nature 393, 152-155 (1998).

3. Steinmetz, N. F. et al. Site-specific and spatially controlled addressability of a new viral nanobuilding block: sulfolobus islandicus rod-shaped virus 2. Adv. Funct. Mater. 18, 3478-3486 (2008).

4. Li, F. \& Wang, Q. Fabrication of nanoarchitectures templated by virus-based nanoparticles: strategies and applications. Small DOI: 10.1002/smll.201301393.

5. Zahr, O. K. \& Blum, A. S. Solution phase gold nanorings on a viral protein template. Nano Lett. 12, 629-633 (2012).

6. Brasch, M. et al. Encapsulation of phthalocyanine supramolecular stacks into virus-like particles. J. Am. Chem. Soc. 133, 6878-6881 (2011).

7. Li, F. et al. Tunable, discrete, three-dimensional hybrid nanoarchitectures. Angew. Chem. Int. Ed. 50, 4202-4205 (2011).

8. Li, F. et al. Monofunctionalization of protein nanocages. J. Am. Chem. Soc. 133, 20040-20042 (2011).

9. Lucon, J. et al. Use of the interior cavity of the P22 capsid for site-specific initiation of atom-transfer radical polymerization with high-density cargo loading. Nat. Chem. 4, 781-788 (2012).

10. Nam, K. T., Peelle, B. R., Lee, S. W. \& Belcher, A. M. Genetically driven assembly of nanorings based on the M13 virus. Nano Lett. 4, 23-27 (2004).

11. Martinez-Morales, A. A. et al. Synthesis and characterization of iron oxide derivatized mutant cowpea mosaic virus hybrid nanoparticles. Adv. Mater. 20, 4816-4820 (2008).

12. Li, F. et al. Three-dimensional gold nanoparticle clusters with tunable cores templated by a viral protein scaffold. Small 8, 3832-3838 (2012).

13. Lewis, J. D. et al. Viral nanoparticles as tools for intravital vascular imaging. Nat. Med. 12, 354-360 (2006).

14. Lee, L. A., Niu, Z. W. \& Wang, Q. Viruses and virus-like protein assemblieschemically programmable nanoscale building blocks. Nano Res. 2, 349-364 (2009).

15. Dixit, S. K. et al. Quantum dot encapsulation in viral capsids. Nano Lett. 6 , 1993-1999 (2006). 
16. Brunel, F. M. et al. Hydrazone ligation strategy to assemble multifunctional viral nanoparticles for cell imaging and tumor targeting. Nano Lett. 10, 1093-1097 (2010).

17. Patterson, D. P., Prevelige, P. E. \& Douglas, T. Nanoreactors by programmed enzyme encapsulation inside the capsid of the bacteriophage P22. Acs Nano 6, 5000-5009 (2012).

18. Shen, L., Bao, N., Zhou, Z., Prevelige, P. E. \& Gupta, A. Materials design using genetically engineered proteins. J. Mater. Chem. 21, 18868-18876 (2011).

19. Li, F. et al. Insights into stabilization of a viral protein cage in templating complex nanoarchitectures: roles of disulfide bonds. Small DOI: 10.1002/smll.201300860.

20. Wang, Q., Lin, T., Tang, L., Johnson, J. E. \& Finn, M. G. Icosahedral virus particles as addressable nanoscale building blocks. Angew. Chem. Int. Edit. 41, 459-462 (2002).

21. Capehart, S. L., Coyle, M. P., Glasgow, J. E. \& Francis, M. B. Controlled integration of gold nanoparticles and organic fluorophores using synthetically modified MS2 viral capsids. J. Am. Chem. Soc. 135, 3011-3016 (2013).

22. Kale, A., Bao, Y., Zhou, Z., Prevelige, P. E. \& Gupta, A. Directed self-assembly of CdS quantum dots on bacteriophage P22 coat protein templates. Nanotechnology 24, 045603 (2013)

23. Glasgow, J. E., Capehart, S. L., Francis, M. B. \& Tullman-Ercek, D. Osmolytemediated encapsulation of proteins inside MS2 viral capsids. ACS Nano 6 , 8658-8664 (2012)

24. Dujardin, E., Peet, C., Stubbs, G., Culver, J. N. \& Mann, S. Organization of metallic nanoparticles using tobacco mosaic virus templates. Nano Lett. 3, 413-417 (2003).

25. Stephanopoulos, N. et al. Immobilization and one-dimensional arrangement of virus capsids with nanoscale precision using DNA origami. Nano Lett. 10, 2714-2720 (2010).

26. Qazi, S. et al. P22 viral capsids as nanocomposite high-relaxivity MRI contrast agents. Mol. Pharmaceut 10, 11-17 (2013).

27. Kang, S., Uchida, M., O’Neil, A., Li, R., Prevelige, P. E. \& Douglas, T. Implementation of P22 viral capsids as nanoplatforms. Biomacromolecules 11, 2804-2809 (2010)

28. Shen, L., Bao, N., Prevelige, P. E. \& Gupta, A. Fabrication of ordered nanostructures of sulfide nanocrystal assemblies over self-assembled genetically engineered P22 coat protein. J. Am. Chem. Soc. 132, 17354-17357 (2010).

29. Parker, M. H. \& Prevelige, P. E. Electrostatic interactions drive scaffolding/coat protein binding and procapsid maturation in bacteriophage P22. Virology 250, 337-349 (1998).

30. Mao, C. et al. Viral assembly of oriented quantum dot nanowires. Proc. Natl. Acad. Sci. U. S. A. 100, 6946-6951 (2003).

31. Mao, C. et al. Virus-based toolkit for the directed synthesis of magnetic and semiconducting nanowires. Science 303, 213-217 (2004).

32. Prevelige, P. E., Thomas, D. \& King, J. Scaffolding protein regulates the polymerization of P22 coat subunits into icosahedral shells in vitro. J. Mol. Biol. 202, 743-757 (1988)

33. Prevelige, P. E., Thomas, D. \& King, J. Nucleation and growth phases in the polymerization of coat and scaffolding subunits into icosahedral procapsid shells. Biophys. J. 64, 824-835 (1993).

34. Reichhardt, C., Uchida, M., O’Neil, A., Li, R., Prevelige, P. E. \& Douglas, T. Templated assembly of organic-inorganic materials using the core shell structure of the P22 bacteriophage. Chem. Commun. 47, 6326-6328 (2011).

35. Flynn, C. E. et al. Synthesis and organization of nanoscale II-VI semiconductor materials using evolved peptide specificity and viral capsid assembly. J. Mater. Chem. 13, 2414-2421 (2003).

36. Tuma, R. et al. A helical coat protein recognition domain of the bacteriophage P22 scaffolding protein. J. Mol. Biol. 281, 81-94 (1998).

37. Parker, M. H., Jablonsky, M., Casjens, S., Sampson, L., Krishna, N. R. \& Prevelige, P. E. Cloning, purification, and preliminary characterization by circular dichroism and NMR of a carboxyl-terminal domain of the bacteriophage P22 scaffolding protein. Protein Sci. 6, 1583-1586 (1997).

38. Bao, N., Shen, L., Takata, T. \& Domen, K. Self-templated synthesis of nanoporous $\mathrm{CdS}$ nanostructures for highly efficient photocatalytic hydrogen production under visible light. Chem. Mater. 20, 110-117 (2008).

39. Bao, N., Shen, L., Lu, X., Yanagisawa, K. \& Feng, X. Room-temperature syntheses of CdS nanocrystals templated by triblock copolymer in aqueous solution under air condition. Chem. Phys. Lett. 377, 119-124 (2003).

40. Parker, M. H., Brouillette, C. G. \& Prevelige, P. E. Kinetic and calorimetric evidence for two distinct scaffolding protein binding populations within the bacteriophage P22 procapsid. Biochemistry 40, 8962-8970 (2001).

41. Padilla-Meier, G. P. \& Teschke, C. M. Conformational changes in bacteriophage P22 scaffolding protein induced by interaction with coat protein. J. Mol. Biol. 410, 226-240 (2011).

42. Gebauer, D., Voelkel, A. \& Coelfen, H. Stable prenucleation calcium carbonate clusters. Science 322, 1819-1822 (2008).

43. Pouget, E. M., Bomans, P. H. H., Dey, A., Frederik, P. M., de With, G. \& Sommerdijk, N. A. J. M. The development of morphology and structure in hxagonal vaterite. J. Am. Chem. Soc. 132, 11560-11565 (2010).
44. Pouget, E. M., Bomans, P. H. H., Goos, J. A. C. M., Frederik, P. M., de With, G. \& Sommerdijk, N. A. J. M. The initial stages of template-controlled $\mathrm{CaCO}_{3}$ formation revealed by Cryo-TEM. Science 323, 1455-1458 (2009).

45. Dey, A. et al. The role of prenucleation clusters in surface-induced calcium phosphate crystallization. Nat. Mater. 9, 1010-1014 (2010).

46. Gebauer, D. \& Coelfen, H. Prenucleation clusters and non-classical nucleation. Nano Today 6, 564-584 (2011).

47. Karan, S. \& Maw, B. Tunable visible-light emission from CdS nanocrystallites prepared under microwave irradiation. J. Phys. Chem. C 111, 16734-16741 (2007).

48. Henglein, A. Small-particle research: physicochemical properties of extremely small colloidal metal and semicondutor particles. Chem. Rev. 89, 1861-1873 (1989).

49. Torimoto, T. et al. Characterization of ultrasmall CdS nanoparticles prepared by the size-selective photoetching technique. J. Phys. Chem. B 105, 6838-6845 (2001).

50. Miyake, M., Torimoto, T., Sakata, T., Mori, H. \& Yoneyama, H. Photoelectrochemical characterization of nearly monodisperse CdS nanoparticles-immobilized gold electrodes. Langmuir 15, 1503-1507 (1999).

51. Yu, W., Qu, L., Guo, W. \& Peng, X. Experimental determination of the extinction coefficient of CdTe, CdSe, and CdS nanocrystals. Chem. Mat. 15, 2854-2860 (2003).

52. Sugimoto, T., Dirige, G. E. \& Muramatsu, A. Formation mechanism of uniform CdS particles from condensed $\mathrm{Cd}(\mathrm{OH})_{2}$ suspension. J. Colloid Interface Sci. 176, 442-453 (1995).

53. Sugimoto, T., Chen, S. \& Muramatsu, A. Synthesis of uniform particles of CdS, $\mathrm{ZnS}, \mathrm{PbS}$ and $\mathrm{CuS}$ from concentrated solutions of the metal chelates. Colloid Surf. A-Physicochem. Eng. Asp. 135, 207-226 (1998).

54. Kubota, N. \& Mullin, J. W. A kinetic model for crystal growth from aqueous solution in the presence of impurity. J. Cryst. Growth 152, 203-208 (1995).

55. Rieger, J., Frechen, T., Cox, G., Heckmann, W., Schmidt, C. \& Thieme, J. Precursor structures in the crystallization/precipitation processes of $\mathrm{CaCO}_{3}$ and control of particle formation by polyelectrolytes. Faraday Discuss. 136, 265-277 (2007).

56. Amjad, Z. Kinetic study of the seeded growth of calcium carbonate in the presence of benzenepolycarboxylic acids. Langmuir 3, 224-228 (1987).

57. Njegic-Dzakula, B., Brecevic, L., Falini, G. \& Kralj, D. Calcite crystal growth kinetics in the presence of charged synthetic polypeptides. Cryst. Growth Des. 9, 2425-2434 (2009).

58. Shen, L., Bao, N., Prevelige, P. E. \& Gupta, A. Escherichia coli bacteria-templated synthesis of nanoporous cadmium sulfide hollow microrods for efficient photocatalytic hydrogen production. J. Phys. Chem. C 114, 2551-2559 (2010).

59. Ma, N., Yang, J., Stewart, K. M. \& Kelley, S. O. DNA-passivated CdS nanocrystals: luminescence, bioimaging, and toxicity profiles. Langmuir 23, 12783-12787 (2007).

60. Santra, S., Yang, H., Holloway, P. H., Stanley, J. T. \& Mericle, R. A. Synthesis of water-dispersible fluorescent, radio-opaque, and paramagnetic CdS: $\mathrm{Mn} / \mathrm{ZnS}$ quantum dots: A multifunctional probe for bioimaging. J. Am. Chem. Soc. 127, 1656-1657 (2005).

\section{Acknowledgments}

This research was supported by the U.S. Department of Energy, Office of Basic Energy Sciences, Division of Materials Sciences and Engineering under Award \# DE-FG02-08ER46537. We thank Dr. Y. Bao and Mr. Y. Xu for the DLS measurements, and Dr. E. Carlson for help with the simulation work.

\section{Author contributions}

Z.Z. performed most of the experiments on CdS, analyzed the data and helped write the manuscript. G.J.B. and R.L. synthesized P22 virus-like particles, P22 coat proteins, and P22 scaffolding proteins, and wrote related synthesis methods. P.E.P. and A.G. conceived the initial hypothesis, designed experiments, analyzed the data and wrote the manuscript.

\section{Additional information}

Supplementary information accompanies this paper at http://www.nature.com/ scientificreports

Competing financial interests: The authors declare no competing financial interests.

How to cite this article: Zhou, Z.Y., Bedwell, G.J., Li, R., Prevelige, P.E. \& Gupta, A. Formation mechanism of chalcogenide nanocrystals confined inside genetically engineered virus-like particles. Sci. Rep. 4, 3832; DOI:10.1038/srep03832 (2014).

This work is licensed under a Creative Commons AttributionNonCommercial-ShareAlike 3.0 Unported license. To view a copy of this license, visit http://creativecommons.org/licenses/by-nc-sa/3.0 